\title{
MORE NOTIONS OF FORCING ADD A SOUSLIN TREE
}

\author{
ARI MEIR BRODSKY AND ASSAF RINOT
}

\begin{abstract}
An $\aleph_{1}$-Souslin tree is a complicated combinatorial object whose existence cannot be decided on the grounds of ZFC alone. But 15 years after Tennenbaum and independently Jech devised notions of forcing for introducing such a tree, Shelah proved that already the simplest forcing notion — Cohen forcing — adds an $\aleph_{1}$-Souslin tree.

In this paper, we identify a rather large class of notions of forcing that, assuming a GCH-type assumption, add a $\lambda^{+}$-Souslin tree. This class includes Prikry, Magidor and Radin forcing.
\end{abstract}

\section{INTRODUCTION}

The definition of a $\kappa$-Souslin tree may be found in Section 2. Our starting point is a theorem of Jensen from his masterpiece [Jen72]. Let $\kappa$ denote a regular uncountable cardinal, and let $E$ denote a stationary subset of $\kappa$. The principle from [Jen72, p. 287], which we denote here by $\square(E)$, asserts the existence of a sequence $\left\langle C_{\alpha} \mid \alpha<\kappa\right\rangle$ such that for every limit ordinal $\alpha<\kappa$ :

- $C_{\alpha}$ is a club in $\alpha$;

- if $\bar{\alpha}$ is an accumulation point of $C_{\alpha}$, then $C_{\alpha} \cap \bar{\alpha}=C_{\bar{\alpha}}$ and $\bar{\alpha} \notin E .{ }^{1}$

The principle from [Jen72, p. 293], commonly denoted $\diamond(E)$, asserts the existence of a sequence $\left\langle Z_{\alpha} \mid \alpha<\kappa\right\rangle$ such that for every subset $Z \subseteq \kappa$, there exist stationarily many $\alpha \in E$ such that $Z \cap \alpha=Z_{\alpha}$. Jensen's theorem reads as follows:

Theorem 1.1 (Jensen, [Jen72, Theorem 6.2]). If $E$ is a stationary subset of a given regular uncountable cardinal $\kappa$, and $\square(E)+\diamond(E)$ holds, then there exists a $\kappa$-Souslin tree.

The goal of this paper is to identify various forcing scenarios that will introduce $\kappa$-Souslin trees. We do so by studying several combinatorial principles that can be used (together with $\diamond(\kappa)$ ) to construct $\kappa$-Souslin trees, and establishing that some forcing scenarios already introduce these. The simplest among the combinatorial principles under consideration is the following:

Definition 1.2. For any regular uncountable cardinal $\kappa, \nabla^{*}(\kappa)$ asserts the existence of a sequence $\left\langle\mathcal{C}_{\alpha} \mid \alpha<\kappa\right\rangle$ such that:

(1) For every limit ordinal $\alpha<\kappa$ :

- $\mathcal{C}_{\alpha}$ is a nonempty collection of club subsets of $\alpha$, with $\left|\mathcal{C}_{\alpha}\right|<\kappa$;

- if $C \in \mathcal{C}_{\alpha}$ and $\bar{\alpha}$ is an accumulation point of $C$, then $C \cap \bar{\alpha} \in \mathcal{C}_{\bar{\alpha}}$;

(2) For every cofinal $A \subseteq \kappa$, there exist stationarily many $\alpha<\kappa$ such that $\sup (\operatorname{nacc}(C) \cap A)=\alpha$ for all $C \in \mathcal{C}_{\alpha}{ }^{2}$

2010 Mathematics Subject Classification. Primary 03E05; Secondary 03E35, 05C05.

Key words and phrases. Souslin-tree construction, microscopic approach, Prikry forcing, Magidor forcing, Radin forcing, parameterized proxy principle, square principle, outside guessing of clubs.

This research was supported by the Israel Science Foundation (grant \#1630/14).

${ }^{1}$ Note that $\square(E)$ for $E=\kappa=\aleph_{1}$ is a trivial consequence of ZFC, and that $\square(E)$ for $\sup (E)=\kappa>\aleph_{1}$ implies that $E \neq \kappa$. Therefore, our choice of notation does not conflict with the principle $\square(\kappa)$ from [Tod87, p. 267].

${ }^{2}$ Here, nacc $(C)$ stands for the set of non-accumulation points of $C$. See the Notation subsection below. 
An evident difference between the principles $\square(E)$ and $\nabla^{*}(\kappa)$ is that the former assigns only a single club to each level $\alpha$, whereas the latter assigns many (like in Jensen's weak square principle). A more substantial difference is that the principle $\square(E)$ implies that the stationary set $E$ is nonreflecting, whereas the principle $\nabla^{*}(\kappa)$ is consistent with the statement that all stationary subsets of $\kappa$ reflect (by [LH16], or by a combination of the main results of [FH16] and [Rin16]).

Nevertheless, $\square^{*}(\kappa)$ is a nontrivial principle. For instance, one can use the function $\rho_{2}$ from the theory of walks on ordinals [Tod87] to show that $\nabla^{*}(\kappa)$ entails the existence of a $\kappa$-Aronszajn tree. More importantly, we have the following:

Proposition A. If $\kappa$ is a regular uncountable cardinal, and $\nabla^{*}(\kappa)+\diamond(\kappa)$ holds, then there exists a $\kappa$-Souslin tree.

Now, there is an obvious way of introducing $\nabla^{*}(\kappa)$ by forcing. Conditions are sequences $\left\langle\mathcal{C}_{\alpha}\right|$ $\alpha \leq \delta\rangle$ of successor length below $\kappa$, such that for every limit ordinal $\alpha \leq \delta$, the two bullets of Definition 1.2(1) hold. But is there another way?

The main result of this paper is the identification of a ceratin class of notions of forcing that (indirectly) introduce $\bigotimes^{*}(\kappa)$.

Definition 1.3. For a regular uncountable cardinal $\lambda$, let $\mathbb{C}_{\lambda}$ denote the class of all notions of forcing $\mathbb{P}$ satisfying the two items:

(1) $\mathbb{P}$ is $\lambda^{+}-c c$ and has size $\leq 2^{\lambda}$;

(2) in $V^{\mathbb{P}}$, there exists a cofinal subset $\Lambda \subseteq \lambda$ such that for every function $f \in{ }^{\lambda} \lambda \cap V$, there exists some $\xi \in \Lambda$ with $f(\xi)<\min (\Lambda \backslash(\xi+1))$.

Clearly, assuming a GCH-type assumption, the various notions of forcing for adding a fast club to $\lambda$ (such as [DJ74, p. 97] and the minor variations [AS83, p. 650], [Rin09, p. 820]) belong to this class. Also, for infinite regular cardinals $\theta<\lambda=\lambda^{<\theta}$, the Lévy collapse $\operatorname{Col}(\theta, \lambda)$ belongs to $\mathbb{C}_{\lambda}$. The next proposition provides considerably more.

Proposition B. Suppose that $\lambda$ is a regular uncountable cardinal satisfying $2^{\lambda}=\lambda^{+}$.

If $\mathbb{P}$ is a $\lambda^{+}$-cc notion of forcing of size $\leq 2^{\lambda}$, then each of the following implies that $\mathbb{P} \in \mathbb{C}_{\lambda}$ :

- $\mathbb{P}$ preserves the regularity of $\lambda$, and is not ${ }^{\lambda} \lambda$-bounding; ${ }^{3}$

- $\mathbb{P}$ forces that $\lambda$ is a singular cardinal;

- $\mathbb{P}$ forces that $\lambda$ is a singular ordinal satisfying $2^{\operatorname{cf}(\lambda)}<\lambda$.

It follows that Cohen, Prikry, Magidor and Radin forcing, ${ }^{4}$ as well as some of the Namba-like forcings from the recent paper by Adolf, Apter, and Koepke [AAK16], are all members of the class under consideration.

Main Theorem. Suppose that $\lambda=\lambda^{<\lambda}$ is a regular uncountable cardinal satisfying $2^{\lambda}=\lambda^{+}$.

Let $\kappa:=\lambda^{+}$. For all $\mathbb{P} \in \mathbb{C}_{\lambda}, V^{\mathbb{P}} \models \nabla^{*}(\kappa)+\diamond(\kappa)$.

One of the aspects that makes the proof of the preceding somewhat difficult is the fact that we do not assume that $\mathbb{P}$ is cofinality-preserving, let alone assume that $\lambda$ remains a regular cardinal in $V^{\mathbb{P}}$. In anticipation of constructions of $\kappa$-Souslin trees of a more involved nature, we will actually want to obtain a stronger principle than $\nabla^{*}(\kappa)$, which we denote by $\mathrm{P}^{*}(T, \lambda)$. This principle dictates a strong form of Clause (2) of Definition 1.2, in which it can be shown that all but nonstationarily

\footnotetext{
${ }^{3}$ Recall that a notion of forcing is ${ }^{\lambda} \lambda$-bounding if for every $g \in{ }^{\lambda} \lambda \cap V^{\mathbb{P}}$, there exists some $f \in{ }^{\lambda} \lambda \cap V$ such that $g(\alpha) \leq f(\alpha)$ for all $\alpha<\lambda$.

${ }^{4}$ This includes variations likes the supercompact Prikry forcing and supercompact Magidor forcing (that singularizes a successor cardinal $\lambda$ ), and includes the instances of Radin forcing that preserves the inaccessibility of $\lambda$.
} 
many $\alpha \in T$ will satisfy $|C|=|\lambda|$ for all $C \in \mathcal{C}_{\alpha}$. When combined with the welcomed possibility that $\mathbb{P}$ forces that $\lambda$ is a singular cardinal (in which case $V^{\mathbb{P}} \models \lambda^{<\lambda}>\lambda$ ), ensuring Clause (1) of Definition 1.2 becomes a burden.

The definition of the weak square principle $\square_{\lambda}^{*}$ may be found in [Jen72, p. 283]. Whether $\mathrm{GCH}+\square_{\lambda}^{*}$ is consistent with the nonexistence of $\lambda^{+}$-Souslin trees for a singular cardinal $\lambda$ is an open problem (see [Sch05]). In [BR16], generalizing a theorem of Gregory from [Gre76], we have shown that any model of such a consistency would have to satisfy that all stationary subsets of $E_{\neq \operatorname{cf}(\lambda)}^{\lambda^{+}}$ reflect. Now, by [CFM01, §11] and [CS02, §4], if GCH holds and $\lambda$ is a $\lambda^{+}$-supercompact cardinal, then in the generic extension by Prikry forcing (using a normal measure on $\lambda$ ), GCH $+\square_{\lambda}^{*}$ holds,

and every stationary subset of $E_{\neq \mathrm{cf}(\lambda)}^{\lambda^{+}}$reflects. The next corollary (which follows from Propositions A,B and the Main Theorem) implies that nevertheless, this model contains a $\lambda^{+}$-Souslin tree.

Corollary A. Suppose that $\lambda$ is a strongly inaccessible cardinal satisfying $2^{\lambda}=\lambda^{+}$.

If $\mathbb{P}$ is a $\lambda^{+}$-cc notion of forcing of size $\leq 2^{\lambda}$ that makes $\lambda$ into a singular cardinal, then $\mathbb{P}$ introduces a $\lambda^{+}$-Souslin tree.

Another corollary to the main result is the following: ${ }^{5}$

Corollary B. Suppose that $\theta<\lambda=\lambda^{<\lambda}$ are infinite regular cardinals, and $2^{\lambda}=\lambda^{+}$.

Then $\operatorname{Col}(\theta, \lambda)$ introduces a $|\lambda|^{+}$-Souslin tree.

At the end of this short paper we shall also quickly deal with the case that $\kappa$ is a former inaccessible:

Proposition C. Suppose that $\lambda^{<\lambda}=\lambda$ is an infinite cardinal, and $\kappa>\lambda$ is a strongly inaccessible cardinal. If $\mathbb{P}$ is a $(<\lambda)$-distributive, $\kappa$-cc notion of forcing, collapsing $\kappa$ to $\lambda^{+}$, then $V^{\mathbb{P}} \models \nabla^{*}(\kappa)+$ $\diamond(\kappa)$ holds.

Examples of notions of forcing satisfying the above requirements include the Lévy collapse $\operatorname{Col}(\lambda,<\kappa)$, and the Silver collapse $\mathbb{S}(\lambda,<\kappa)$.

Notation. For an infinite cardinal $\lambda$, write $\mathrm{CH}_{\lambda}$ for the assertion that $2^{\lambda}=\lambda^{+}$. Next, suppose that $C, D$ are sets of ordinals. Write $\operatorname{acc}(C):=\{\alpha \in C \mid \sup (C \cap \alpha)=\alpha>0\}, \operatorname{nacc}(C):=C \backslash \operatorname{acc}(C)$, and $\operatorname{acc}^{+}(C):=\{\alpha<\sup (C) \mid \sup (C \cap \alpha)=\alpha>0\}$. For any $j<\operatorname{otp}(C)$, denote by $C(j)$ the unique element $\delta \in C$ for which otp $(C \cap \delta)=j$. For any ordinal $\sigma$, write $\operatorname{succ}_{\sigma}(C):=\{C(j+1) \mid$ $j<\sigma \& j+1<\operatorname{otp}(C)\}$. Write $D \sqsubseteq C$ iff there exists some ordinal $\beta$ such that $D=C \cap \beta$. Write $D \sqsubseteq{ }^{*} C$ iff there exists $\gamma<\sup (D)$ such that $D \backslash \gamma \sqsubseteq C \backslash \gamma$. Write $D \chi \sqsubseteq C$ iff $((D \sqsubseteq C)$ or $(\operatorname{cf}(\sup (D))<\chi))$, and write $D \chi \sqsubseteq^{*} C$ iff $\left(\left(D \sqsubseteq^{*} C\right)\right.$ or $\left.(\operatorname{cf}(\sup (D))<\chi)\right)$.

Acknowledgment. We thank Gitik and Karagila for illuminating discussions on [Git95] and [DS95].

\section{A construction of a Souslin tree from a WeAK hypothesis}

We begin this section by recalling the terminology relevant to trees and fixing some notation.

A tree is a partially ordered set $\left(T,<_{T}\right)$ with the property that for every $x \in T$, the downward cone $x_{\downarrow}:=\left\{y \in T \mid y<_{T} x\right\}$ is well-ordered by $<_{T}$. The height of $x \in T$, denoted ht $(x)$, is the order-type of $\left(x_{\downarrow},<_{T}\right)$. Then, the $\alpha^{\text {th }}$ level of $\left(T,<_{T}\right)$ is the set $T_{\alpha}:=\{x \in T \mid \operatorname{ht}(x)=\alpha\}$. We also write $T \nmid X:=\{t \in T \mid \operatorname{ht}(t) \in X\}$. A tree $\left(T,<_{T}\right)$ is said to be $\chi$-complete if any $<_{T}$-increasing sequence of elements from $T$, and of length $<\chi$, has an upper bound in $T$. A tree $\left(T,<_{T}\right)$ is said

\footnotetext{
${ }^{5}$ But note that Proposition 3.6 yields a stronger conclusion from a weaker arithmetic assumption.
} 
to be normal if for all ordinals $\alpha<\beta$ and every $x \in T_{\alpha}$, if $T_{\beta} \neq \emptyset$ then there exists some $y \in T_{\beta}$ such that $x<_{T} y$. A tree $\left(T,<_{T}\right)$ is said to be splitting if every node in $T$ admits at least two immediate successors.

Let $\kappa$ denote a regular uncountable cardinal. A tree $\left(T,<_{T}\right)$ is a $\kappa$-tree whenever $\left\{\alpha \mid T_{\alpha} \neq \emptyset\right\}=$ $\kappa$, and $\left|T_{\alpha}\right|<\kappa$ for all $\alpha<\kappa$. A subset $B \subseteq T$ is a cofinal branch if $\left(B,<_{T}\right)$ is linearly ordered and $\{\mathrm{ht}(t) \mid t \in B\}=\{\mathrm{ht}(t) \mid t \in T\}$. A $\kappa$-Aronszajn tree is a $\kappa$-tree with no cofinal branches. A $\kappa$-Souslin tree is a $\kappa$-Aronszajn tree that has no antichains of size $\kappa$.

A $\kappa$-tree is said to be binary if it is a downward-closed subset of the complete binary tree ${ }^{<\kappa} 2$, ordered by $\subset$.

All the combinatorial principles considered in this paper are simplified instances of the proxy principle $\mathrm{P}^{-}(\kappa, \mu, \mathcal{R}, \theta, \mathcal{S}, \nu, \sigma, \mathcal{E})$ that was introduced and studied in [BR15a],[BR16], but familiarity with those papers is not needed.

Definition 2.1. Suppose that $\kappa$ is a regular uncountable cardinal, and $S$ is a stationary subset of $\kappa$. Let $\chi:=\min \{\operatorname{cf}(\alpha) \mid \alpha \in S$ limit $\}$. The principle $\nabla^{*}(S)$ asserts the existence of a sequence $\left\langle\mathcal{C}_{\alpha} \mid \alpha<\kappa\right\rangle$ such that:

(1) For every limit ordinal $\alpha<\kappa$ :

- $\mathcal{C}_{\alpha}$ is a nonempty collection of club subsets of $\alpha$, with $\left|\mathcal{C}_{\alpha}\right|<\kappa$;

- if $C \in \mathcal{C}_{\alpha}$ and $\bar{\alpha}$ is an accumulation point of $C$, then there exists some $D \in \mathcal{C}_{\bar{\alpha}}$ satisfying $D_{\chi} \sqsubseteq^{*} C$;

(2) For every cofinal $A \subseteq \kappa$, there exist stationarily many $\alpha \in S$ such that $\sup (\operatorname{nacc}(C) \cap A)=\alpha$ for all $C \in \mathcal{C}_{\alpha}$.

Remarks. i. While it is not entirely obvious, we omit the proof of the fact that $\nabla^{*}(S)$ for $S=\kappa$ coincides with Definition 1.2 given in the previous section.

ii. Note that in general, it is not the case that $\nabla^{*}(S)$ implies $\nabla^{*}(T)$ for $T \supseteq S$.

iii. By [Tod87, Theorem 1.8], if $\kappa$ is a weakly compact cardinal, then $\otimes^{*}(S)$ fails for every stationary subset $S \subseteq \kappa$.

iv. In the langauge of [BR15a],[BR16], $\nabla^{*}(S)$ stands for $\mathrm{P}^{-}\left(\kappa, \kappa, \chi \sqsubseteq^{*}, 1,\{S\}, \kappa, 1, \mathcal{E}_{\kappa}\right)$. In particular, by $[\mathrm{BR} 15 \mathrm{a}], \boldsymbol{\otimes}^{*}\left(E_{\geq \chi}^{\kappa}\right)+\diamond(\kappa)$ is consistent together with $\neg \diamond\left(E_{\geq \chi}^{\kappa}\right)$.

Proposition A is a special case of the following:

Proposition 2.2. If $\kappa$ is a regular uncountable cardinal and $\chi<\kappa$ is a cardinal satisfying $\lambda^{<\chi}<\kappa$ for all $\lambda<\kappa$, then $\nabla^{*}\left(E_{\geq \chi}^{\kappa}\right)+\diamond(\kappa)$ entails a normal, binary, splitting, $\chi$-complete $\kappa$-Souslin tree.

Proof. In [BR15a, §2], we provided a construction of a $\chi$-complete $\kappa$-Souslin using the stronger hypothesis $\nabla^{-}\left(E_{\geq \chi}^{\kappa}\right)$. Here we show that by taking some extra care in the construction, we can get by assuming merely $\otimes^{*}\left(E_{\geq \chi}^{\kappa}\right)$.

Let $\overrightarrow{\mathcal{C}}=\left\langle\mathcal{C}_{\alpha} \mid \alpha<\kappa\right\rangle$ be a witness to $\nabla^{*}\left(E_{\geq \chi}^{\kappa}\right)$. By [BR15a, 2 ],$\diamond(\kappa)$ is equivalent to $\diamond\left(H_{\kappa}\right)$, meaning that, in particular, we may fix a sequence $\left\langle\Omega_{\beta} \mid \beta<\kappa\right\rangle$ satisfying the following: For every $\Omega \subseteq H_{\kappa}$ and $p \in H_{\kappa^{+}}$, there exists an elementary submodel $\mathcal{M} \prec H_{\kappa^{+}}$containing $p$, such that $\mathcal{M} \cap \kappa \in \kappa$ and $\mathcal{M} \cap \Omega=\Omega_{\mathcal{M} \cap \kappa}$.

Let $\triangleleft$ be some well-ordering of $H_{\kappa}$. We shall recursively construct a sequence $\left\langle T_{\alpha} \mid \alpha<\kappa\right\rangle$ of levels whose union will ultimately be the desired tree $T$.

Let $T_{0}:=\{\emptyset\}$, and for all $\alpha<\kappa$, let $T_{\alpha+1}:=\left\{t^{\frown}\langle 0\rangle, t^{\frown}\langle 1\rangle \mid t \in T_{\alpha}\right\}$.

Next, suppose that $\alpha<\kappa$ is a nonzero limit ordinal, and that $\left\langle T_{\beta} \mid \beta<\alpha\right\rangle$ has already been defined. Constructing the level $T_{\alpha}$ involves deciding which branches through $(T \uparrow \alpha, \subset)$ will have 
their limits placed into the tree. We need $T_{\alpha}$ to contain enough nodes to ensure that the tree is normal, but we have to define $T_{\alpha}$ carefully, so that the resulting tree doesn't include large antichains.

Construction of the level $T_{\alpha}$ splits into two cases, depending on the value of $\operatorname{cf}(\alpha)$ :

- If $\operatorname{cf}(\alpha)<\chi$, let $T_{\alpha}$ consist of the limits of all branches through $(T\lceil\alpha, \subset)$. This construction ensures that the tree will be $\chi$-complete, and as any branch through $(T\lceil\alpha, \subset)$ is determined by a subset of $T \uparrow \alpha$ of cardinality $\operatorname{cf}(\alpha)$, the arithmetic hypothesis ensures that $\left|T_{\alpha}\right|<\kappa$ at these levels. Normality at these levels is verified by induction: Fixing a sequence of ordinals of minimal order-type converging to $\alpha$ enables us to find, for any given $x \in T\lceil\alpha$, a branch through $(T\lceil\alpha, \subset)$ containing $x$, and the limit of such a branch will necessarily be in $T_{\alpha}$.

- Now suppose $\operatorname{cf}(\alpha) \geq \chi$. Considering any $C \in \mathcal{C}_{\alpha}$, the idea for ensuring normality at level $T_{\alpha}$ is to attach to each node $x \in T \uparrow C$ some node $\mathbf{b}_{x}^{C}: \alpha \rightarrow 2$ above it, and then let

$$
T_{\alpha}:=\left\{\mathbf{b}_{x}^{C} \mid C \in \mathcal{C}_{\alpha}, x \in T\lceil C\} .\right.
$$

By the induction hypothesis, $\left|T_{\beta}\right|<\kappa$ for all $\beta<\alpha$, and by the choice of $\overrightarrow{\mathcal{C}}$, we have $\left|\mathcal{C}_{\alpha}\right|<\kappa$, so that we are guaranteed to end up with $\left|T_{\alpha}\right|<\kappa$.

Let $C \in \mathcal{C}_{\alpha}$ and $x \in T\left\lceil C\right.$ be arbitrary. As $\mathbf{b}_{x}^{C}$ will be the limit of some branch through $(T\lceil\alpha, \subset)$ and above $x$, it makes sense to describe $\mathbf{b}_{x}^{C}$ as the limit $\bigcup \operatorname{Im}\left(b_{x}^{C}\right)$ of a sequence $b_{x}^{C} \in \prod_{\beta \in C \backslash \operatorname{dom}(x)} T_{\beta}$ such that:

- $b_{x}^{C}(\operatorname{dom}(x))=x$;

- $b_{x}^{C}\left(\beta^{\prime}\right) \subset b_{x}^{C}(\beta)$ for all $\beta^{\prime}<\beta$ in $(C \backslash \operatorname{dom}(x))$;

- $b_{x}^{C}(\beta)=\bigcup \operatorname{Im}\left(b_{x}^{C} \uparrow \beta\right)$ for all $\beta \in \operatorname{acc}(C \backslash \operatorname{dom}(x))$.

Of course, we have to define $b_{x}^{C}$ carefully, so that the resulting tree doesn't include large antichains. We do this by recursion:

Let $b_{x}^{C}(\operatorname{dom}(x)):=x$. Next, suppose $\beta^{-}<\beta$ are consecutive points of $(C \backslash \operatorname{dom}(x))$, and $b_{x}^{C}\left(\beta^{-}\right) \in T_{\beta^{-}}$has already been identified. In order to decide $b_{x}^{C}(\beta)$, we advise with the following set:

Now, consider the two possibilities:

$$
Q_{x}^{C, \beta}:=\left\{t \in T_{\beta} \mid \exists s \in \Omega_{\beta}\left[\left(s \cup b_{x}^{C}\left(\beta^{-}\right)\right) \subseteq t\right]\right\} .
$$

- If $Q_{x}^{C, \beta} \neq \emptyset$, then let $b_{x}^{C}(\beta)$ be its $\triangleleft$-least element;

- Otherwise, let $b_{x}^{C}(\beta)$ be the $\triangleleft$-least element of $T_{\beta}$ that extends $b_{x}^{C}\left(\beta^{-}\right)$. Such an element must exist, as the level $T_{\beta}$ was constructed so as to preserve normality.

Finally, suppose $\beta \in \operatorname{acc}(C \backslash \operatorname{dom}(x))$ and $b_{x}^{C} \uparrow \beta \in \prod_{\delta \in C \cap \beta \backslash \operatorname{dom}(x)} T_{\delta}$ has already been defined. As promised, we let $b_{x}^{C}(\beta):=\bigcup \operatorname{Im}\left(b_{x}^{C} \uparrow \beta\right)$. It is clear that $b_{x}^{C}(\beta) \in{ }^{\beta} 2$, but we need more than that:

Claim 2.2.1. $b_{x}^{C}(\beta) \in T_{\beta}$.

Proof. We consider two cases, depending on the value of $\operatorname{cf}(\beta)$ :

- If $\operatorname{cf}(\beta)<\chi$, then $T_{\beta}$ was constructed to consist of the limits of all branches through $(T \uparrow \beta, \subset)$, including the limit of the branch $b_{x}^{C} \uparrow \beta$, which is $b_{x}^{C}(\beta)$.

- Now suppose $\operatorname{cf}(\beta) \geq \chi$. In this case, since $\beta \in \operatorname{acc}(C)$ and by $\chi \sqsubseteq^{*}$-coherence of the $\nabla^{*}\left(E_{\geq \chi}^{\kappa}\right)$ sequence $\overrightarrow{\mathcal{C}}$, we can pick some $D \in \mathcal{C}_{\beta}$ such that $D \sqsubseteq^{*} C$, and we can thus fix some $\gamma \in(C \cap$ $D) \backslash \operatorname{dom}(x)$ such that $D \backslash \gamma=C \cap \beta \backslash \gamma$. Put $d:=D \backslash \gamma$ and $y:=b_{x}^{C}(\gamma)$. Then $y \in T_{\gamma}$ and $\operatorname{dom}\left(b_{y}^{D}\right)=d=C \cap \beta \backslash \gamma=\operatorname{dom}\left(b_{x}^{C}\right) \cap \beta \backslash \gamma$.

It suffices to prove that $b_{x}^{C} \uparrow d=b_{y}^{D}$, as this will imply that $b_{x}^{C}(\beta)=\bigcup \operatorname{Im}\left(b_{y}^{D}\right)=\mathbf{b}_{y}^{D} \in T_{\beta}$, since the limit of a branch is determined by any of its cofinal segments. Thus, we prove by induction that for every $\delta \in d$, the value of $b_{y}^{D}(\delta)$ was determined in exactly the same way as $b_{x}^{C}(\delta)$ : 
- Clearly, $b_{y}^{D}(\min (d))=y=b_{x}^{C}(\min (d))$, since $\min (d)=\gamma=\operatorname{dom}(y)$.

- Suppose $\delta^{-}<\delta$ are successive points of $d$. Notice that the definition of $Q_{x}^{C, \delta}$ depends only on $b_{x}^{C}\left(\delta^{-}\right), \Omega_{\delta}$, and $T_{\delta}$, and so if $b_{x}^{C}\left(\delta^{-}\right)=b_{y}^{D}\left(\delta^{-}\right)$, then $Q_{x}^{C, \delta}=Q_{y}^{D, \delta}$, and hence $b_{x}^{C}(\delta)=b_{y}^{D}(\delta)$.

- For $\delta \in \operatorname{acc}(d)$ : If the sequences are identical up to $\delta$, then their limits must be identical.

This completes the definition of $b_{x}^{C}$ for each $C \in \mathcal{C}_{\alpha}$ and each $x \in T \uparrow C$, and hence of the level $T_{\alpha}$.

Having constructed all levels of the tree, we then let

$$
T:=\bigcup_{\alpha<\kappa} T_{\alpha} .
$$

Notice that for every $\alpha<\kappa, T_{\alpha}$ is a subset of ${ }^{\alpha} 2$ of size $<\kappa$. Altogether, $(T, \subset)$ is a normal, binary, splitting, $\chi$-complete $\kappa$-tree.

Our next task is proving that $(T, \subset)$ is $\kappa$-Souslin. As any splitting $\kappa$-tree with no antichains of size $\kappa$ also has no chains of size $\kappa$, it suffices to prove Claim 2.2.3 below. For this, we shall need the following:

Claim 2.2.2. Suppose that $A \subseteq T$ is a maximal antichain. Then the set

$$
B:=\left\{\beta<\kappa \mid A \cap(T \uparrow \beta)=\Omega_{\beta} \text { is a maximal antichain in } T \uparrow \beta\right\} .
$$

is a stationary subset of $\kappa$.

Proof. Let $D \subseteq \kappa$ be an arbitrary club. We must show that $D \cap B \neq \emptyset$. Put $p:=\{A, T, D\}$ and $\Omega:=A$. By our choice of the sequence $\left\langle\Omega_{\beta} \mid \beta<\kappa\right\rangle$, pick $\mathcal{M} \prec H_{\kappa^{+}}$with $p \in \mathcal{M}$ such that $\beta:=\mathcal{M} \cap \kappa$ is in $\kappa$ and $\Omega_{\beta}=\mathcal{M} \cap A$. Since $D \in \mathcal{M}$ and $D$ is club in $\kappa$, we have $\beta \in D$. We claim that $\beta \in B$.

For all $\alpha<\beta$, by $\alpha, T \in \mathcal{M}$, we have $T_{\alpha} \in \mathcal{M}$, and by $\mathcal{M} \models\left|T_{\alpha}\right|<\kappa$, we have $T_{\alpha} \subseteq \mathcal{M}$. So $T \uparrow \beta \subseteq \mathcal{M}$. As $\operatorname{dom}(z) \in \mathcal{M}$ for all $z \in T \cap \mathcal{M}$, we conclude that $T \cap \mathcal{M}=T \uparrow \beta$. Thus, $\Omega_{\beta}=A \cap\left(T\lceil\beta)\right.$. As $H_{\kappa^{+}} \models A$ is a maximal antichain in $T$, it follows by elementarity that $\mathcal{M} \models A$ is a maximal antichain in $T$. Since $T \cap \mathcal{M}=T \uparrow \beta$, we get that $A \cap(T \uparrow \beta)$ is a maximal antichain in $T \uparrow \beta$.

Claim 2.2.3. Suppose that $A \subseteq T$ is a maximal antichain. Then $|A|<\kappa$.

Proof. Let $A \subseteq T$ be a maximal antichain. By Claim 2.2.2,

$$
B:=\left\{\beta<\kappa \mid A \cap(T \uparrow \beta)=\Omega_{\beta} \text { is a maximal antichain in } T \uparrow \beta\right\}
$$

is a stationary subset of $\kappa$. Thus we apply $\nabla^{*}\left(E_{\geq \chi}^{\kappa}\right)$ to obtain an ordinal $\alpha \in E_{\geq \chi}^{\kappa}$ satisfying

$$
\sup (\operatorname{nacc}(C) \cap B)=\alpha
$$

for every $C \in \mathcal{C}_{\alpha}$.

We shall prove that $A \subseteq T\lceil\alpha$, from which it follows that $|A| \leq|T| \alpha \mid<\kappa$.

To see that $A \subseteq T\lceil\alpha$, consider any $z \in T\lceil(\kappa \backslash \alpha)$, and we will show that $z \notin A$ by finding some element of $A \cap(T\lceil\alpha)$ compatible with $z$.

Since $\operatorname{dom}(z) \geq \alpha$, we can let $y:=z\left\lceil\alpha\right.$. Then $y \in T_{\alpha}$ and $y \subseteq z$. By construction, since $\operatorname{cf}(\alpha) \geq \chi$, we have $y=\mathbf{b}_{x}^{C}=\bigcup_{\beta \in C \backslash \operatorname{dom}(x)} b_{x}^{C}(\beta)$ for some $C \in \mathcal{C}_{\alpha}$ and some $x \in T \uparrow C$. Fix $\beta \in \operatorname{nacc}(C) \cap B$ with $\operatorname{dom}(x)<\beta<\alpha$. Denote $\beta^{-}:=\sup (C \cap \beta)$. Then $\beta^{-}<\beta$ are consecutive points of $C \backslash \operatorname{dom}(x)$. Since $\beta \in B$, we know that $\Omega_{\beta}=A \cap(T \uparrow \beta)$ is a maximal antichain in $T\lceil\beta$, and hence there is some $s \in \Omega_{\beta}$ compatible with $b_{x}^{C}\left(\beta^{-}\right)$, so that by normality of the tree, $Q_{x}^{C, \beta} \neq \emptyset$. 
It follows that we chose $b_{x}^{C}(\beta)$ to extend some $s \in \Omega_{\beta}$. Altogether, $s \subseteq b_{x}^{C}(\beta) \subset \mathbf{b}_{x}^{C}=y \subseteq z$. Since $s$ is an element of the antichain $A$, the fact that $z$ extends $s$ implies that $z \notin A$.

So $(T, \subset)$ is a normal, binary, splitting, $\chi$-complete $\kappa$-Souslin tree.

\section{Main Results}

We begin this section by proving Proposition B:

Proposition 3.1. Suppose that $\lambda$ is a regular uncountable cardinal satisfying $2^{\lambda}=\lambda^{+}$. If $\mathbb{P}$ is a $\lambda^{+}$-cc notion of forcing of size $\leq 2^{\lambda}$, then each of the following implies that $\mathbb{P} \in \mathbb{C}_{\lambda}$ :

(1) $\mathbb{P}$ preserves the regularity of $\lambda$, and is not ${ }^{\lambda} \lambda$-bounding;

(2) $\mathbb{P}$ forces that $\lambda$ is a singular cardinal;

(3) $\mathbb{P}$ forces that $\lambda$ is a singular ordinal, satisfying $2^{\mathrm{cf}(\lambda)}<\lambda$.

Proof. Let $G$ denote a $\mathbb{P}$-generic filter.

(1) Work in $V[G]$. Since $\mathbb{P}$ is not ${ }^{\lambda} \lambda$-bounding, let us pick $g \in{ }^{\lambda} \lambda$ with the property that for every $f \in{ }^{\lambda} \lambda \cap V$, there exists some $\alpha<\lambda$ with $f(\alpha)<g(\alpha)$. Since $\lambda$ is regular and uncountable, the set $\Lambda:=\{\zeta<\lambda \mid g[\zeta] \subseteq \zeta\}$ is a club in $\lambda$. To see that $\Lambda$ works, let $f \in{ }^{\lambda} \lambda \cap V$ be arbitrary. In $V$, let $f^{\prime}: \lambda \rightarrow \lambda$ be a strictly increasing function such that $f(\xi) \leq f^{\prime}(\xi)$ for all $\xi<\lambda$. Now, back in $V[G]$, pick $\alpha<\lambda$ such that $f^{\prime}(\alpha)<g(\alpha)$. Put $\xi:=\sup (\Lambda \cap(\alpha+1))$ and $\zeta:=\min (\Lambda \backslash(\xi+1))$. Then $\xi \in \Lambda$ because $\Lambda$ is a club in $\lambda$ containing 0 , and $f(\xi) \leq f^{\prime}(\xi) \leq f^{\prime}(\alpha)<g(\alpha)<\zeta$ because $\alpha<\zeta$ and $\zeta \in \Lambda$.

(2) Note that as $\mathbb{P}$ forces that $\lambda$ is a singular cardinal, $\lambda$ cannot be a successor cardinal in the ground model. Also note that by the $\lambda^{+}-c c$ of $\mathbb{P}$, we have $\left(\lambda^{+}\right)^{V[G]}=\left(\lambda^{+}\right)^{V}$. Thus, by [DS95, Theorem 2.0], since $\lambda$ is inaccessible, $\left(\lambda^{+}\right)^{V[G]}=\left(\lambda^{+}\right)^{V}$ and $2^{\lambda}=\lambda^{+}$, there exists a cofinal $\Lambda \subseteq \lambda$ in $V[G]$ such that $\sup (\Lambda \backslash C)<\lambda$ for every club $C$ in $\lambda$ from $V$. Thus, to see that $\Lambda$ works, let $f \in{ }^{\lambda} \lambda \cap V$ be arbitrary. Consider the club $C:=\{\zeta<\lambda \mid f[\zeta] \subseteq \zeta\}$. As $C \in V$, pick $\xi \in \Lambda$ such that $\Lambda \backslash \xi \subseteq C$. Put $\zeta:=\min (\Lambda \backslash(\xi+1))$. Then $\zeta \in C$ and hence $f(\xi)<\zeta$.

(3) Let $\kappa:=\left(\lambda^{+}\right)^{V}$. Work in $V[G]$. Let $\theta:=\operatorname{cf}(\lambda)$. By $2^{\theta}<\lambda$, we have $\left(2^{\theta}\right)^{+} \leq|\lambda|^{+}$. Since $\mathbb{P}$ is $\kappa$-cc, we have $|\lambda|^{+}=\kappa$. In particular, $\operatorname{cf}(\kappa)=\kappa \geq\left(2^{\theta}\right)^{+}$. Also, by $2^{\theta}<\lambda$, we have $\theta^{+}<\lambda$. Then, the two conditions of [Git95, Proposition 2.1] are satisfied and hence there exists a cofinal $\Lambda \subseteq \lambda$ such that $\sup (\Lambda \backslash C)<\lambda$ for every club $C$ in $\lambda$ from $V$. Thus, we are done as in the previous case.

Lemma 3.2. Suppose that $\lambda$ is a regular uncountable cardinal, and $\mathbb{P} \in \mathbb{C}_{\lambda}$.

In $V^{\mathbb{P}}$, there exists a club $\Lambda \subseteq \lambda$ of order-type $\operatorname{cf}(\lambda)$, such that for every function $f \in{ }^{\lambda} \lambda \cap V$, $\sup \{\xi \in \Lambda \mid f(\xi)<\min (\Lambda \backslash(\xi+1))\}=\lambda$.

Proof. Let $G$ be $\mathbb{P}$-generic, and work in $V[G]$. Pick $\Lambda$ as in Clause (2) of Definition 1.3. Let $\Lambda$ be a club in $\lambda$ of order-type $\operatorname{cf}(\lambda)$ such that $\operatorname{nacc}(\Lambda) \subseteq \Lambda$. To see that $\Lambda$ works, fix an arbitrary $f \in{ }^{\lambda} \lambda \cap V$ and an arbitrary $\iota<\lambda$. We shall find $\dot{\xi} \in \AA_{\Lambda}^{\circ}$ above $\iota$ satisfying $f(\xi)<\min (\Lambda \backslash(\dot{\xi}+1))$.

Fix a large enough $\epsilon \in \operatorname{nacc}(\Lambda)$ such that $\sup (\Lambda \cap \epsilon)>\iota$. In $V$, using the regularity of $\lambda$, pick a strictly increasing function $f^{\prime}: \lambda \rightarrow \lambda$ such that $f^{\prime}(\alpha) \geq \max \{f(\alpha), \epsilon\}$ for all $\alpha<\lambda$. By the choice of $\Lambda$, let us pick $\xi \in \Lambda$ such that $f^{\prime}(\xi)<\min (\Lambda \backslash(\xi+1))$. In particular, $\epsilon<\zeta$, where $\zeta:=\min (\Lambda \backslash(\xi+1))$. As $\epsilon \in \operatorname{nacc}(\Lambda) \subseteq \Lambda$, and as $\xi<\zeta$ are two successive elements of $\Lambda$ with

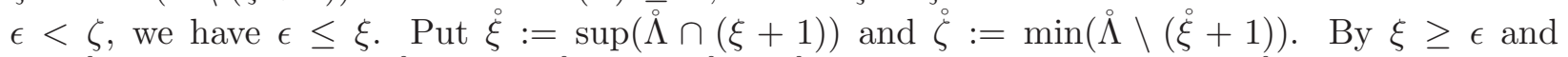
$\sup (\Lambda \cap \epsilon)>\iota$, we have $\stackrel{\xi}{\xi}>\iota$. By $\stackrel{\zeta}{\zeta} \in \operatorname{nacc}(\Lambda) \backslash(\dot{\xi}+1) \subseteq \Lambda \backslash(\xi+1)$, we have $\dot{\zeta} \geq \zeta$. Altogether:

- $\iota<\stackrel{\circ}{\xi} \leq \xi<\zeta \leq \dot{\zeta}$, and 
- $f(\dot{\xi}) \leq f^{\prime}(\stackrel{\circ}{\xi}) \leq f^{\prime}(\xi)<\zeta \leq \stackrel{\circ}{\zeta}=\min (\stackrel{\circ}{\Lambda} \backslash(\stackrel{\circ}{\xi}+1))$

So that $\stackrel{\circ}{\xi}$ is as sought.

Definition 3.3. Suppose that $T$ is a stationary subset of a regular uncountable cardinal $\kappa$, and $\xi \leq \kappa$ is an ordinal.

The principle $\mathrm{P}^{*}(T, \xi)$ asserts the existence of a sequence $\left\langle\mathcal{C}_{\alpha} \mid \alpha<\kappa\right\rangle$ such that:

(1) For every limit ordinal $\alpha<\kappa$ :

- $\mathcal{C}_{\alpha}$ is a nonempty collection of club subsets of $\alpha$ of order-type $\leq \xi$, with $\left|\mathcal{C}_{\alpha}\right|<\kappa$;

- if $C \in \mathcal{C}_{\alpha}$ and $\bar{\alpha}$ is an accumulation point of $C$, then $C \cap \bar{\alpha} \in \mathcal{C}_{\bar{\alpha}}$;

(2) For every cofinal subset $A \subseteq \kappa$, all but nonstationarily many $\alpha \in T$ satisfy:

- $\left|\mathcal{C}_{\alpha}\right|=1$, say, $\mathcal{C}_{\alpha}=\left\{C_{\alpha}\right\}$, and

- $\sup \left\{\beta \in C_{\alpha} \mid \operatorname{succ}_{\sigma}\left(C_{\alpha} \backslash \beta\right) \subseteq A\right\}=\alpha$ for every $\sigma<\operatorname{otp}\left(C_{\alpha}\right)$.

Remarks. i. Note that for stationary sets $S \subseteq T \subseteq \kappa, \mathrm{P}^{*}(T, \xi) \Longrightarrow \mathrm{P}^{*}(S, \xi) \Longrightarrow \mathrm{P}^{*}(S, \kappa) \Longrightarrow$ $\nabla^{*}(S)$, and that $\mathrm{P}^{*}(S, \kappa) \Longrightarrow \mathbb{\nabla}^{*}(\kappa)$. Thus, the remaining theorems in this paper will focus on establishing $\mathrm{P}^{*}(T, \xi)+\diamond(\kappa)$ for some stationary $T \subseteq \kappa$ and some $\xi \leq \kappa$ in various forcing scenarios.

ii. By arguments that may be found in [BR15b, §6], $\mathrm{P}^{*}(S, \kappa)+\diamond(\kappa)$ entails the existence of a $\kappa$-Souslin tree which is moreover free. We do not know whether $\nabla^{*}(\kappa)+\diamond(\kappa)$ suffices for this application.

iii. In the langauge of $[\mathrm{BR} 15 \mathrm{a}],[\mathrm{BR} 16], \mathrm{P}^{*}(T, \xi)$ stands for $\mathrm{P}^{-}\left(\kappa, \kappa, \sqsubseteq, 1, \mathrm{NS}_{\kappa} \uparrow T, 2,<\infty, \mathcal{E}_{\xi}\right)$.

We now arrive at the main result of this paper:

Theorem 3.4. Suppose that $\lambda^{<\lambda}=\lambda$ is a regular uncountable cardinal, and $\mathrm{CH}_{\lambda}$ holds.

Let $\kappa:=\lambda^{+}$and $T:=E_{\lambda}^{\kappa}$. For every $\mathbb{P} \in \mathbb{C}_{\lambda}, V^{\mathbb{P}} \models \mathrm{P}^{*}(T, \lambda)+\diamond(\kappa)$.

Proof. By $\mathrm{CH}_{\lambda}$ and the main result of [She10], $\diamond(\kappa)$ holds. By [BR15a, 2 ],$\diamond(\kappa)$ is equivalent to $\diamond\left(H_{\kappa}\right)$, meaning that, in particular, we may fix a sequence $\left\langle\Omega_{\beta} \mid \beta<\kappa\right\rangle$ satisfying the following: For every $\Omega \subseteq H_{\kappa}$ and $p \in H_{\kappa^{+}}$, there exists an elementary submodel $\mathcal{M} \prec H_{\kappa^{+}}$containing $p$, such that $\mathcal{M} \cap \kappa \in \kappa$ and $\mathcal{M} \cap \Omega=\Omega_{\mathcal{M} \cap \kappa}$.

Let $\mathbb{P} \in \mathbb{C}_{\lambda}$ be arbitrary. By $|\mathbb{P}| \leq\left|H_{\kappa}\right|$, we may assume that $\mathbb{P} \subseteq H_{\kappa}$. Let $G \subseteq \mathbb{P}$ be $V$-generic, and work in $V[G]$. Since $\mathbb{P}$ is a $\kappa-c c$ notion of forcing, $\kappa$ remains a regular cardinal and $T$ remains stationary in $V[G]$.

For all $\beta<\kappa$ such that $\Omega_{\beta}$ happens to be a $\mathbb{P}$-name for a subset of $\beta$, let $Z_{\beta}$ denote its interpretation by $G$. For all other $\beta<\kappa$, let $Z_{\beta}:=\emptyset$.

Claim 3.4.1. For every $A \in \mathcal{P}^{V[G]}(\kappa)$, there exists some $X_{A} \in \mathcal{P}^{V}(\kappa)$ such that:

(1) $V \models X_{A}$ is stationary;

(2) $V[G] \models X_{A} \subseteq\left\{\beta<\kappa \mid Z_{\beta}=A \cap \beta\right\}$.

Proof. Fix an arbitrary $A \subseteq \kappa$ in $V[G]$, and let $\dot{A}$ be a $\mathbb{P}$-name for $A$.

Work in $V$. For every $\alpha<\kappa$, put $O_{\alpha}:=\left\{p \in \mathbb{P} \mid p \Vdash_{\mathbb{P}} \check{\alpha} \in \dot{A}\right\}$, and choose some maximal antichain $A_{\alpha} \subseteq O_{\alpha}$. Then $\Omega:=\bigcup\left\{\{\check{\alpha}\} \times A_{\alpha} \mid \alpha<\kappa\right\}$ is a nice name for $A$. In particular:

- $A_{\alpha}$ is an antichain in $\mathbb{P}$ for all $\alpha<\kappa$;

- $\vdash_{\mathbb{P}} \dot{A}=\Omega$.

Since $\mathbb{P} \subseteq H_{\kappa}$ is a $\kappa$-cc notion of forcing, we also have:

- $A_{\alpha} \in H_{\kappa}$ for all $\alpha<\kappa$. 
Altogether, $\Omega \subseteq H_{\kappa},|\Omega| \leq \kappa$, and hence $\Omega \in H_{\kappa^{+}}$. Let

$$
X_{A}:=\left\{\mathcal{M} \cap \kappa \mid \mathcal{M} \prec H_{\kappa^{+}}, \Omega \in \mathcal{M}, \mathcal{M} \cap \kappa \in \kappa, \mathcal{M} \cap \Omega=\Omega_{\mathcal{M} \cap \kappa}\right\} .
$$

(1) To see that $X_{A}$ is stationary, let $D$ be an arbitrary club in $\kappa$. Put $p:=\{\Omega, D\}$. By the fixed witness to $\diamond\left(H_{\kappa}\right)$, we may now pick an elementary submodel $\mathcal{M} \prec H_{\kappa^{+}}$such that $p \in \mathcal{M}$, $\mathcal{M} \cap \kappa \in \kappa$ and $\mathcal{M} \cap \Omega=\Omega_{\mathcal{M} \cap \kappa}$. Denote $\beta:=\mathcal{M} \cap \kappa$. By $D \in \mathcal{M}$, we have $\beta \in D \cap X_{A}$.

(2) Let $\beta \in X_{A}$ be arbitrary, with witnessing model $\mathcal{M}$. By $\Omega \in \mathcal{M}$ and $\left|A_{\alpha}\right|<\kappa$ for all $\alpha<\kappa$, we have $\Omega_{\beta}=\mathcal{M} \cap \Omega=\bigcup\left\{\{\check{\alpha}\} \times A_{\alpha} \mid \alpha<\beta\right\}$. So $\Omega_{\beta}$ is a nice name and $\Vdash_{\mathbb{P}} \Omega_{\beta}=\Omega \cap \check{\beta}=\dot{A} \cap \check{\beta}$. That is, $\Omega_{\beta}$ is a $\mathbb{P}$-name whose interpretation in $V[G]$ is $A \cap \beta$, and hence $V[G] \models Z_{\beta}=A \cap \beta$.

Since $\mathbb{P}$ is a $\kappa$-cc notion of forcing, every stationary subset of $\kappa$ from $V$ remains stationary in $V[G]$, and so it follows from the previous claim that $\diamond(\kappa)$ holds in $V[G]$. Thus, we are left with verifying that $\mathrm{P}^{*}(T, \lambda)$ holds in $V[G]$.

Work back in $V$. By $\lambda^{<\lambda}=\lambda$, let us fix a sequence of injections $\left\langle\varrho_{\alpha}: \alpha \rightarrow \lambda \mid \alpha<\kappa\right\rangle$ with the property that for all $\delta<\kappa$, we have $\left|\left\{\varrho_{\alpha}|\delta| \alpha<\kappa\right\}\right|<\kappa{ }^{6}$. For every $\alpha \in T$, let $\pi_{\alpha}: \lambda \rightarrow E_{<\lambda}^{\alpha}$ denote the monotone enumeration of some club in $\alpha$. Notice that $\operatorname{Im}\left(\pi_{\alpha}\right) \cap T=\emptyset$ for every $\alpha \in T$.

By $\mathrm{CH}_{\lambda}$, let us fix an enumeration $\left\{X^{j} \mid j<\kappa\right\}$ of all bounded subsets of $\kappa$ such that each element appears cofinally often.

Next, for every nonempty $x \in[\kappa]^{<\lambda}$ and every $\beta \in x \cap T$, define $\left.h_{x, \beta}: \operatorname{otp}(x) \rightarrow[\beta]^{1} \cup[\beta]^{(1+o t p}(x)\right)$ as follows:

Put $h_{x, \beta}(0):=\{\sup (x \cap \beta)\}$. Now, if $i \in(0, \operatorname{otp}(x))$ and $h_{x, \beta}\lceil i$ has already been defined, write $\epsilon_{x, \beta}(i):=\sup \left(\bigcup_{i^{\prime}<i} h_{x, \beta}\left(i^{\prime}\right)\right)+1$ and $j:=x(i)$. Then:

- If $\sup \left(X^{j} \cap \beta\right) \neq \beta$, then let $h_{x, \beta}(i):=\left\{\epsilon_{x, \beta}(i)\right\} ;$

- If $\sup \left(X^{j} \cap \beta\right)=\beta$, then let $h_{x, \beta}(i):=\left\{\epsilon_{x, \beta}(i)\right\} \cup \operatorname{succ}_{\text {otp }(x)}\left(X^{j} \backslash \epsilon_{x, \beta}(i)\right)$.

Note that all of the above are well-defined, thanks to the fact that $\operatorname{cf}(\beta)=\lambda>|x|$.

Finally, for every nonempty $x \in[\kappa]^{<\lambda}$, let $\bar{x}$ be the ordinal closure of

$$
x \cup \bigcup\left\{\bigcup \operatorname{Im}\left(h_{x, \beta}\right) \mid \beta \in x \cap T, \beta \neq \min (x)\right\} .
$$

Clearly, $\min (\bar{x})=\min (x), \max (\bar{x})=\sup (x)$, and $|\bar{x}| \leq \max \left\{|x|, \aleph_{0}\right\}$. In particular, otp $(\bar{x})<\lambda$. In addition, for all $\beta \in x \cap T$ such that $\gamma:=\sup (x \cap \beta)$ is nonzero, we have that $\bar{x} \cap[\gamma, \beta)$ is equal to the ordinal closure of $\bigcup \operatorname{Im}\left(h_{x, \beta}\right)$.

Work in $V[G]$. By $\mathbb{P} \in \mathbb{C}_{\lambda}$, let $\Lambda$ be the club given by Lemma 3.2. For all $\alpha \in T$, let:

$$
C_{\alpha}:=\bigcup\left\{\overline{\left[\pi_{\alpha}(\xi), \pi_{\alpha}(\zeta)\right) \cap\left(\left\{\pi_{\alpha}(\xi)\right\} \cup \varrho_{\alpha}^{-1}[\zeta]\right)} \mid \xi \in \Lambda, \zeta=\min (\Lambda \backslash(\xi+1))\right\} .
$$

Notice that $\pi_{\alpha}[\Lambda] \subseteq C_{\alpha} \subseteq \alpha$, so that $C_{\alpha}$ is a club in $\alpha$.

Before stating the next claim, let us remind the reader that we do not assume that $\mathbb{P}$ is cofinalitypreserving.

Claim 3.4.2. For every $\alpha \in T$ :

(1) $\operatorname{acc}\left(C_{\alpha}\right) \cap T=\emptyset$;

(2) $\operatorname{otp}\left(C_{\alpha}\right) \leq \lambda$.

\footnotetext{
${ }^{6}$ Note that the existence of such a sequence is equivalent to the existence of a special $\lambda^{+}$-Aronszajn tree, which is a well-known consequence of $\lambda^{<\lambda}=\lambda$ (cf. [Spe49] or [Tod84, Theorem 7.1]).
} 
Proof. (1) Suppose that $\bar{\alpha} \in \operatorname{acc}\left(C_{\alpha}\right)$. Put $\xi^{\prime}:=\sup \left\{\xi \in \Lambda \mid \pi_{\alpha}(\xi) \leq \bar{\alpha}\right\}$.

- If $\bar{\alpha}=\pi_{\alpha}\left(\xi^{\prime}\right)$, then $\bar{\alpha} \in \operatorname{Im}\left(\pi_{\alpha}\right)$ and hence $\bar{\alpha} \notin T$.

- Otherwise, letting $\zeta^{\prime}:=\min \left(\Lambda \backslash\left(\xi^{\prime}+1\right)\right)$, we get that $\bar{\alpha}$ is an accumulation point of

$$
\overline{\left[\pi_{\alpha}\left(\xi^{\prime}\right), \pi_{\alpha}\left(\zeta^{\prime}\right)\right) \cap\left(\left\{\pi_{\alpha}\left(\xi^{\prime}\right)\right\} \cup \varrho_{\alpha}^{-1}\left[\zeta^{\prime}\right]\right)},
$$

which is a set of ordinals from $V$ of size $<\lambda$, and hence $\operatorname{cf}^{V}(\bar{\alpha})<\lambda$, so that $\bar{\alpha} \notin T$.

(2) We prove by induction on $\xi \in \Lambda$ that $\operatorname{otp}\left(C_{\alpha} \cap \pi_{\alpha}(\xi)\right)<\lambda$ for all $\xi \in \Lambda$ :

- For $\xi=\min (\Lambda)$, we have $C_{\alpha} \cap \pi_{\alpha}(\xi)=\emptyset$.

- Suppose that $\xi \in \Lambda$ and $\operatorname{otp}\left(C_{\alpha} \cap \pi_{\alpha}(\xi)\right)<\lambda$. Put $\zeta:=\min (\Lambda \backslash(\xi+1))$. Then

$$
\operatorname{otp}\left(C_{\alpha} \cap \pi_{\alpha}(\zeta)\right)=\operatorname{otp}\left(C_{\alpha} \cap \pi_{\alpha}(\xi)\right)+\operatorname{otp}\left(\overline{\left[\pi_{\alpha}(\xi), \pi_{\alpha}(\zeta)\right) \cap\left(\left\{\pi_{\alpha}(\xi)\right\} \cup \varrho_{\alpha}^{-1}[\zeta]\right)}\right),
$$

so that $\operatorname{otp}\left(C_{\alpha} \cap \pi_{\alpha}(\zeta)\right)$ is the sum of two ordinals $<\lambda$. As $\lambda$ is a cardinal in $V$, it is an additively indecomposable ordinal, and hence the sum of the two is still $<\lambda$.

- Suppose that $\xi \in \operatorname{acc}(\Lambda)$ and $\operatorname{otp}\left(C_{\alpha} \cap \pi_{\alpha}(\epsilon)\right)<\lambda$ for all $\epsilon \in \Lambda \cap \xi$. That is,

$$
\left\{\operatorname{otp}\left(C_{\alpha} \cap \pi_{\alpha}(\epsilon)\right) \mid \epsilon \in \Lambda \cap \xi\right\} \subseteq \lambda .
$$

As $\operatorname{otp}\left(C_{\alpha} \cap \pi_{\alpha}(\xi)\right)=\sup \left\{\operatorname{otp}\left(C_{\alpha} \cap \pi_{\alpha}(\epsilon)\right) \mid \epsilon \in \Lambda \cap \xi\right\}$, and $|\Lambda \cap \xi|<|\Lambda|=\operatorname{cf}(\lambda)$, we infer that $\operatorname{otp}\left(C_{\alpha} \cap \pi_{\alpha}(\xi)\right)<\lambda$.

Claim 3.4.3. For every $\delta<\kappa$, we have $\left|\left\{C_{\alpha} \cap \delta \mid \alpha \in T\right\}\right|<\kappa$.

Proof. Suppose not, and let $\delta$ be the least counterexample. Pick a subset $A \subseteq T \backslash(\delta+1)$ of size $\kappa$ such that:

- $\alpha \mapsto C_{\alpha} \cap \delta$ is injective over $A$;

- $\alpha \mapsto \varrho_{\alpha}\lceil\delta$ is constant over $A$;

- $\alpha \mapsto \sup \left\{\xi \in \Lambda \mid \pi_{\alpha}(\xi) \leq \delta\right\}$ is constant over $A$, with value, say, $\xi^{\prime}$;

- $\alpha \mapsto \pi_{\alpha}\left\lceil\left(\xi^{\prime}+1\right)\right.$ is constant over $A .^{7}$

Clearly, $\alpha \mapsto C_{\alpha} \cap \pi_{\alpha}\left(\xi^{\prime}\right)$ is constant over $A$. Write $\zeta^{\prime}:=\min \left(\Lambda \backslash\left(\xi^{\prime}+1\right)\right)$. Then it must be the case that $\alpha \mapsto C_{\alpha} \cap\left[\pi_{\alpha}\left(\xi^{\prime}\right), \delta\right)$ is injective over $A$. That is,

$$
\alpha \mapsto \overline{\left[\pi_{\alpha}\left(\xi^{\prime}\right), \pi_{\alpha}\left(\zeta^{\prime}\right)\right) \cap\left(\left\{\pi_{\alpha}\left(\xi^{\prime}\right)\right\} \cup \varrho_{\alpha}^{-1}\left[\zeta^{\prime}\right]\right)} \cap \delta
$$

is injective over $A$, contradicting the fact that the right-hand side of the above mapping is an element of $[\delta]^{<\lambda} \cap V$, and $V \models\left|[\delta]^{<\lambda}\right|<\kappa$.

Since $\mathbb{P}$ is a $\kappa$-cc notion of forcing, every club $D \subseteq \kappa$ from $V[G]$ contains a club $D^{\prime} \subseteq D$ from $V$. Hence, whenever we talk about clubs in $\kappa$, we may as well assume that they lie in $V$.

Claim 3.4.4. For every cofinal subset $X \subseteq \kappa$ from $V$, there exists a club $D_{X} \subseteq \kappa$, such that for every $\alpha \in T \cap D_{X}$ and every $\sigma<\lambda$, we have $\sup \left\{\epsilon \in C_{\alpha} \mid \operatorname{succ}_{\sigma}\left(C_{\alpha} \backslash \epsilon\right) \subseteq X\right\}=\alpha$.

Proof. Work in $V$. Given a cofinal subset $X \subseteq \kappa$, define $f: \kappa \rightarrow \kappa$ by stipulating

$$
f(\beta):=\min \left\{j<\kappa \mid X^{j}=X \cap \beta \& j \geq \beta\right\} .
$$

Consider the club $D_{X}:=\{\alpha<\kappa \mid f[\alpha] \subseteq \alpha\} \cap \operatorname{acc}^{+}\left(\operatorname{acc}^{+}(X) \cap T\right)$. Let $\alpha \in T \cap D_{X}$ be arbitrary. Let $\tau<\alpha$ and $\sigma<\lambda$ be arbitrary. We shall prove the existence of some $\epsilon \in C_{\alpha} \backslash \tau$ such that $\operatorname{succ}_{\sigma}\left(C_{\alpha} \backslash \epsilon\right) \subseteq X$.

\footnotetext{
${ }^{7}$ Here, we use two facts: (1) If $\pi_{\alpha}\left(\xi^{\prime}\right) \leq \delta$, then $\pi_{\alpha} \uparrow\left(\xi^{\prime}+1\right)$ is the increasing enumeration of an element of $[\delta+1]^{<\lambda} \cap V$. (2) By $V \models\left|[\delta+1]^{<\lambda}\right|=\lambda<\kappa$, we have $V[G] \models\left|[\delta+1]^{<\lambda} \cap V\right|<\kappa$.
} 
Let $\varrho_{\alpha}^{-01}: \lambda \rightarrow \alpha$ denote a pseudoinverse of the injection $\varrho_{\alpha}: \alpha \rightarrow \lambda$, as follows:

$$
\varrho_{\alpha}^{-01}(\xi):= \begin{cases}\varrho_{\alpha}^{-1}(\xi), & \text { if } \xi \in \operatorname{Im}\left(\varrho_{\alpha}\right) \\ 0, & \text { otherwise. }\end{cases}
$$

Define $f_{0}, f_{1}, f_{2}, f_{3}: \lambda \rightarrow \lambda$ by stipulating:

- $f_{0}(\xi):=\min \left(\varrho_{\alpha}\left[\left(\operatorname{acc}^{+}(X) \cap T \cap \alpha\right) \backslash \pi_{\alpha}(\xi)\right]\right)$;

- $f_{1}(\xi):=\varrho_{\alpha}\left(f\left(\varrho_{\alpha}^{-01}(\xi)\right)\right)$;

- $f_{2}(\xi):=\min \left\{\zeta<\lambda \mid \varrho_{\alpha}^{-01}(\xi) \leq \pi_{\alpha}(\zeta)\right\}$

- $f_{3}(\xi):=\min \left\{\zeta<\lambda \mid \operatorname{otp}\left(\varrho_{\alpha}^{-1}[\zeta] \cap\left[\pi_{\alpha}(\xi), \pi_{\alpha}(\zeta)\right) \geq \sigma\right\}\right.$.

$f_{0}$ is well-defined since $\alpha \in \operatorname{acc}^{+}\left(\operatorname{acc}^{+}(X) \cap T\right) . f_{1}$ is well-defined since $f[\alpha] \subseteq \alpha . f_{2}$ is well-defined since $\operatorname{Im}\left(\pi_{\alpha}\right)$ is cofinal in $\alpha . f_{3}$ is well-defined since $\operatorname{cf}(\alpha)=\lambda$, so that $\operatorname{otp}\left(\left[\pi_{\alpha}(\xi), \alpha\right)\right) \geq \lambda>\sigma$ for all $\xi<\lambda$.

Define $f_{*}: \lambda \rightarrow \lambda$ by stipulating:

$$
f_{*}(\xi):=\max \left\{f_{0}(\xi), f_{1}\left(f_{0}(\xi)\right), f_{2}\left(f_{1}\left(f_{0}(\xi)\right)\right), f_{3}(\xi)\right\} .
$$

From now on, work in $V[G]$. By the choice of $\Lambda$, pick a large enough $\xi \in \Lambda$ such that $f_{*}(\xi)<$ $\min (\Lambda \backslash(\xi+1))$ and $\pi_{\alpha}(\xi)>\tau$. Denote $\zeta:=\min (\Lambda \backslash(\xi+1))$. Clearly, $C_{\alpha} \cap\left[\pi_{\alpha}(\xi), \pi_{\alpha}(\zeta)\right)=\bar{x}$, where

$$
x:=\left[\pi_{\alpha}(\xi), \pi_{\alpha}(\zeta)\right) \cap\left(\left\{\pi_{\alpha}(\xi)\right\} \cup \varrho_{\alpha}^{-1}[\zeta]\right) .
$$

Put $\zeta_{0}:=f_{0}(\xi), \zeta_{1}:=f_{1}\left(\zeta_{0}\right), \zeta_{2}:=f_{2}\left(\zeta_{1}\right)$, and $\zeta_{3}:=f_{3}(\xi)$. Evidently, $\zeta_{0}, \zeta_{1} \in \operatorname{Im}\left(\varrho_{\alpha}\right)$. By $f_{*}(\xi)<\zeta$, we have $\zeta_{i}<\zeta$ for all $i<4$.

Write $\beta:=\varrho_{\alpha}^{-1}\left(\zeta_{0}\right), j:=f(\beta)$ and $\gamma:=\sup (x \cap \beta)$. Then $\beta \in T, j=\varrho_{\alpha}^{-1}\left(\zeta_{1}\right)$, and $X^{j}=X \cap \beta$ is a cofinal subset of $\beta$. As $\zeta_{2}<\zeta, \min (x)=\pi_{\alpha}(\xi) \notin T$ and $\beta \in T \backslash \pi_{\alpha}(\xi)$, we infer that

$$
\tau<\pi_{\alpha}(\xi) \leq \gamma<\beta \leq j \leq \pi_{\alpha}\left(\zeta_{2}\right)<\pi_{\alpha}(\zeta)<\alpha .
$$

By $\zeta_{0}<\zeta$ and $\zeta_{1}<\zeta$, we altogether have $\{\beta, j\} \subseteq x \backslash\{\min (x)\}$. Fix some nonzero $i<\operatorname{otp}(x)$ such that $j=x(i)$. Recalling that $X^{j}=X \cap \beta$ is a cofinal subset of $\beta$, we have:

$$
h_{x, \beta}(i)=\left\{\epsilon_{x, \beta}(i)\right\} \cup \operatorname{succ}_{\mathrm{otp}(x)}\left(X \cap \beta \backslash \epsilon_{x, \beta}(i)\right),
$$

where $\operatorname{otp}\left(h_{x, \beta}(i)\right)=1+\operatorname{otp}(x)$ and $h_{x, \beta}(i) \subseteq(\gamma, \beta)$.

As $f_{3}(\xi)=\zeta_{3}<\zeta$, we have otp $(x) \geq \sigma$, and so in particular

$$
\operatorname{succ}_{\sigma}\left(\bigcup \operatorname{Im}\left(h_{x, \beta}\right) \backslash \epsilon_{x, \beta}(i)\right) \subseteq X .
$$

As $\beta \in x \cap T, \beta \neq \min (x)$, and $\gamma=\sup (x \cap \beta)$, we know that $C_{\alpha} \cap[\gamma, \beta)=\bar{x} \cap[\gamma, \beta)$ is equal to the ordinal closure of $\bigcup \operatorname{Im}\left(h_{x, \beta}\right)$. Put $\epsilon:=\epsilon_{x, \beta}(i)$. Then $\epsilon \in C_{\alpha} \cap(\gamma, \beta) \subseteq C_{\alpha} \backslash \tau$, and $\operatorname{succ}_{\sigma}\left(C_{\alpha} \backslash \epsilon\right) \subseteq X$, as sought.

For every $\alpha \in T$, let $C_{\alpha}^{\bullet}:=\operatorname{Im}\left(g_{\alpha}\right)$, where $g_{\alpha}: C_{\alpha} \rightarrow \alpha$ is defined by stipulating:

$$
g_{\alpha}(\beta):= \begin{cases}\beta, & \text { if } \beta \in \operatorname{acc}\left(C_{\alpha}\right) ; \\ \min \left(Z_{\beta} \cup\{\beta\}\right), & \text { if } \beta=\min \left(C_{\alpha}\right) ; \\ \min \left(\left(Z_{\beta} \cup\{\beta\}\right) \backslash\left(\sup \left(C_{\alpha} \cap \beta\right)+1\right)\right), & \text { otherwise. }\end{cases}
$$

Note that $C_{\alpha}^{\bullet}(i) \leq C_{\alpha}(i)<C_{\alpha}^{\bullet}(i+1)$ for all $i<\operatorname{otp}\left(C_{\alpha}\right)$. So $g_{\alpha}$ is strictly increasing and continuous, and $C_{\alpha}^{\bullet}$ is a club subset of $\alpha$ with $\operatorname{otp}\left(C_{\alpha}^{\bullet}\right)=\operatorname{otp}\left(C_{\alpha}\right) \leq \lambda, \operatorname{acc}\left(C_{\alpha}^{\bullet}\right)=\operatorname{acc}\left(C_{\alpha}\right)$, and $\operatorname{nacc}\left(C_{\alpha}^{\bullet}\right)=g_{\alpha}\left[\operatorname{nacc}\left(C_{\alpha}\right)\right]$.

Claim 3.4.5. For every cofinal subset $A \subseteq \kappa$ (from $V[G]$ ), there exists a club $E_{A} \subseteq \kappa$, such that for every $\alpha \in T \cap E_{A}$ and every $\sigma<\lambda$, we have $\sup \left\{\eta \in C_{\alpha}^{\bullet} \mid \operatorname{succ}_{\sigma}\left(C_{\alpha}^{\bullet} \backslash \eta\right) \subseteq A\right\}=\alpha$. 
Proof. Given a cofinal subset $A \subseteq \kappa$ from $V[G]$, let $X_{A} \in \mathcal{P}^{V}(\kappa)$ be given by Claim 3.4.1. In particular, $V \models X_{A}$ is a stationary subset of $\kappa$. As $V[G] \models \operatorname{acc}^{+}(A)$ is a club in $\kappa$, and $V[G]$ is a $\kappa$-cc forcing extension of $V$, we may fix some $B \in V$ such that $V \models B$ is a club in $\kappa$, and $V[G] \models B \subseteq \operatorname{acc}^{+}(A)$. Put $X:=X_{A} \cap B$. Then:

(1) $V \models X$ is stationary in $\kappa$;

(2) $V[G] \models X \subseteq\left\{\beta<\kappa \mid Z_{\beta}=A \cap \beta\right.$ is a cofinal subset of $\left.\beta\right\}$.

Now, let $E_{A}:=D_{X}$, where $D_{X}$ is given by Claim 3.4.4. Let $\alpha \in T \cap E_{A}$ and $\sigma<\lambda$ be arbitrary. Then $\sup \left\{\epsilon \in C_{\alpha} \mid \operatorname{succ}_{\sigma}\left(C_{\alpha} \backslash \epsilon\right) \subseteq X\right\}=\alpha$. To see that $\sup \left\{\eta \in C_{\alpha}^{\bullet} \mid \operatorname{succ}_{\sigma}\left(C_{\alpha}^{\bullet} \backslash \eta\right) \subseteq A\right\}=\alpha$, let $\tau<\alpha$ be arbitrary. Choose $\epsilon \in C_{\alpha}$ such that $\operatorname{succ}_{\sigma}\left(C_{\alpha} \backslash \epsilon\right) \subseteq X$ and $g_{\alpha}(\epsilon)>\tau$. Let $\eta:=g_{\alpha}(\epsilon)$. To see that $\operatorname{succ}_{\sigma}\left(C_{\alpha}^{\bullet} \backslash \eta\right) \subseteq A$, consider an arbitrary $i<\sigma$, and we will show that $\left(C_{\alpha}^{\bullet} \backslash \eta\right)(i+1) \in A$.

Write $\beta:=\left(C_{\alpha} \backslash \epsilon\right)(i+1)$. Then $\beta \in \operatorname{nacc}\left(C_{\alpha}\right)$, and $\beta \in X$, so that $Z_{\beta}=A \cap \beta$ is a cofinal subset of $\beta$, and hence

$\left(C_{\alpha}^{\bullet} \backslash \eta\right)(i+1)=g_{\alpha}(\beta)=\min \left(\left(Z_{\beta} \cup\{\beta\}\right) \backslash\left(\sup \left(C_{\alpha} \cap \beta\right)+1\right)\right)=\min \left(A \backslash\left(\sup \left(C_{\alpha} \cap \beta\right)+1\right)\right) \in A$, as required.

Let $\mathcal{C}_{0}^{\bullet}:=\{\emptyset\}$, and $\mathcal{C}_{\delta+1}^{\bullet}:=\{\{\delta\}\}$ for all $\delta<\kappa$. For every $\delta \in T$, let $\mathcal{C}_{\delta}^{\bullet}:=\left\{C_{\delta}^{\bullet}\right\}$, and for every $\delta \in \operatorname{acc}(\kappa) \backslash T$, let

$$
\mathcal{C}_{\delta}^{\bullet}:=\left\{C_{\alpha}^{\bullet} \cap \delta \mid \alpha \in T, \sup \left(C_{\alpha}^{\bullet} \cap \delta\right)=\delta\right\} \cup\left\{c \in[\delta]^{<\lambda} \cap V \mid c \text { is a club in } \delta\right\} .
$$

Claim 3.4.6. $\left|\mathcal{C}_{\delta}^{\bullet}\right|<\kappa$ for all $\delta<\kappa$.

Proof. Suppose not, and let $\delta \in \operatorname{acc}(\kappa) \backslash T$ be a counterexample. As $V \models\left|[\delta]^{<\lambda}\right| \leq \lambda<\kappa$, and by Claim 3.4.3, let us pick $\alpha<\alpha^{\prime}$ both from $T$ and above $\delta$ such that $C_{\alpha}^{\bullet} \cap \delta \neq C_{\alpha^{\prime}}^{\bullet} \cap \delta$, $\sup \left(C_{\alpha}^{\bullet} \cap \delta\right)=\delta=\sup \left(C_{\alpha^{\prime}}^{\bullet} \cap \delta\right)$, and $C_{\alpha} \cap \delta=C_{\alpha^{\prime}} \cap \delta$. By $\delta \in \operatorname{acc}\left(C_{\alpha}^{\bullet}\right)$, we have $g_{\alpha}(\delta)=\delta$, and hence $C_{\alpha}^{\bullet} \cap \delta=g_{\alpha}[\delta]$. Likewise, $C_{\alpha^{\prime}}^{\bullet} \cap \delta=g_{\alpha^{\prime}}[\delta]$. So $g_{\alpha} \uparrow \delta \neq g_{\alpha^{\prime}} \uparrow \delta$, contradicting the fact that $C_{\alpha} \cap \delta=C_{\alpha^{\prime}} \cap \delta$.

Altogether, $\left\langle\mathcal{C}_{\delta}^{\bullet} \mid \delta<\kappa\right\rangle$ witnesses $\mathrm{P}^{*}(T, \lambda)$.

Corollary 3.5. Suppose that $\lambda$ is a strongly inaccessible cardinal satisfying $2^{\lambda}=\lambda^{+}$.

If $\mathbb{P}$ is a $\lambda^{+}$-cc notion of forcing of size $\leq 2^{\lambda}$ that makes $\lambda$ into a singular cardinal, then $\mathbb{P}$ introduces a free $\lambda^{+}$-Souslin tree.

Proof. By Proposition 3.1, $\mathbb{P} \in \mathbb{C}_{\lambda}$. By Theorem 3.4, then, in $V^{\mathbb{P}}, \mathrm{P}^{*}(T, \lambda)+\diamond\left(\lambda^{+}\right)$holds for some stationary subset $T$ of $\lambda^{+}$.

Finally, by [BR16], $\mathrm{P}^{*}(T, \lambda)+\diamond\left(\lambda^{+}\right)$entails $\mathrm{P}\left(\lambda^{+}, \lambda^{+}, \sqsubseteq, \lambda^{+},\left\{\lambda^{+}\right\}, 2,1, \mathcal{E}_{\lambda^{+}}\right)$, which by the arguments of $[\mathrm{BR} 15 \mathrm{~b}, \S 6]$ suffices for the construction of a free $\lambda^{+}$-Souslin tree.

By Theorem 3.4, if $\theta<\lambda=\lambda^{<\lambda}$ are infinite regular cardinals, and $\mathrm{CH}_{\lambda}$ holds, then $V^{\operatorname{Col}(\theta, \lambda)} \models$ $\mathrm{P}^{*}(T, \lambda)+\diamond(\kappa)$, where $\kappa:=\lambda^{+}$and $T:=E_{\lambda}^{\kappa}$, provided a fact we have already mentioned but did not prove: $\operatorname{Col}(\theta, \lambda) \in \mathbb{C}_{\lambda}$ in this scenario.

The next proposition shows that moreover $\mathrm{P}^{*}(T,|\lambda|)$ holds in the extension. A byproduct of its proof, will also establish that indeed $\operatorname{Col}(\theta, \lambda) \in \mathbb{C}_{\lambda}$.

Proposition 3.6. Suppose that $\theta<\lambda=\lambda^{<\theta}$ are infinite regular cardinals, and $\mathrm{CH}_{\lambda}$ holds.

Let $\kappa:=\lambda^{+}$and $T:=E_{\lambda}^{\kappa}$. Then $V^{\operatorname{Col}(\theta, \lambda)} \models \mathrm{P}^{*}(T, \theta)+\diamond(\kappa)$.

Proof. Work in $V$. For every nonzero $\alpha<\lambda^{+}$, fix a surjection $f_{\alpha}: \lambda \rightarrow \alpha$. Work in $V[G]$, where $G$ is $\operatorname{Col}(\theta, \lambda)$-generic. Put $g:=\bigcup G$. By genericity, $g: \theta \rightarrow \lambda$ is a shuffling surjection, that is, it satisfies $\left|g^{-1}\{\eta\}\right|=\theta$ for every $\eta<\lambda$. 
Note that as $\operatorname{Col}(\theta, \lambda)$ is $(<\theta)$-closed, for every $\alpha \in T$, we have $\theta \leq \operatorname{cf}(\alpha) \leq|\alpha|=|\lambda|=\theta$. That is, $T \subseteq E_{\theta}^{\kappa}$. Fix $\alpha \in T$. Put $g_{\alpha}:=f_{\alpha} \circ g$. As $g$ is a shuffling surjection, so is $g_{\alpha}: \theta \rightarrow \alpha$. Then, since $\operatorname{cf}(\alpha)=\theta$, we may define a strictly increasing function $h_{\alpha}: \theta \rightarrow \theta$ by recursion:

- $h_{\alpha}(0):=0$;

- $h_{\alpha}(i+1):=\min \left\{k \in\left(h_{\alpha}(i), \theta\right) \mid g_{\alpha}(k)>g_{\alpha}\left(h_{\alpha}(i)\right)\right\}$

- for $i \in \operatorname{acc}(\theta), h_{\alpha}(i):=\min \left\{k \in\left(\sup \left(\operatorname{Im}\left(h_{\alpha}\lceil i)\right), \theta\right) \mid g_{\alpha}(k)=\sup \left(\operatorname{Im}\left(g_{\alpha} \circ\left(h_{\alpha}\lceil i)\right)\right)\right\}\right.\right.$.

Clearly, $C_{\alpha}:=\operatorname{Im}\left(g_{\alpha} \circ h_{\alpha}\right)$ is a subset of $\alpha$ of order-type $\theta$ satisfying $\operatorname{acc}^{+}\left(C_{\alpha}\right) \subseteq C_{\alpha}$. It follows from the next claim (taking $X:=\kappa$ ) that $C_{\alpha}$ is moreover a club in $\alpha$.

Claim 3.6.1. For every cofinal subset $X \subseteq \kappa$ from $V$, every $\alpha \in T \cap \operatorname{acc}^{+}(X)$, and every $\sigma<\theta$, we have $\sup \left\{\epsilon \in C_{\alpha} \mid \operatorname{succ}_{\sigma}\left(C_{\alpha} \backslash \epsilon\right) \subseteq X\right\}=\alpha{ }^{8}$

Proof. Work in $V$. Let $X, \alpha$ and $\sigma$ be as in the hypothesis. Let $\varepsilon<\alpha$ be arbitrary.

To run a density argument, let us fix an arbitrary condition $p \in \operatorname{Col}(\theta, \lambda)$. By extending $p$, we may assume that $\bar{\theta}:=\operatorname{dom}(p)$ is a nonzero ordinal. Put $p_{\alpha}:=f_{\alpha} \circ p$, and let $\chi$ be the largest ordinal for which there exists a strictly increasing function $h: \chi \rightarrow \bar{\theta}$ satisfying:

- $h(0)=0$;

- $h(i+1)=\min \left\{k \in(h(i), \bar{\theta}) \mid p_{\alpha}(k)>p_{\alpha}(h(i))\right\}$;

- for $i \in \operatorname{acc}(\chi), h(i)=\min \left\{k \in\left(\sup (\operatorname{Im}(h\lceil i)), \bar{\theta}) \mid p_{\alpha}(k)=\sup \left(\operatorname{Im}\left(p_{\alpha} \circ(h\lceil i))\right)\right\}\right.\right.$.

Let $\beta:=\sup \left(\operatorname{Im}\left(p_{\alpha} \circ h\right)\right)$, and $\epsilon:=\max \{\beta, \varepsilon\}$. As $\left|\operatorname{Im}\left(p_{\alpha}\right)\right| \leq|\bar{\theta}|<\theta=\operatorname{cf}(\alpha)$, we know that $\epsilon<\alpha$. For all $\iota<\theta$, denote $x_{\iota}:=\{\beta, \varepsilon\} \cup \operatorname{succ}_{\iota}(X \backslash \epsilon)$.

Pick a large enough $\iota<\theta$ such that otp $\left(\operatorname{succ}_{\sigma}\left(x_{\iota} \backslash \epsilon\right)\right)=\sigma$, and let $\bar{x}$ denote the ordinal closure of $x_{\iota}$. By $\iota<\theta$ and $\alpha \in \operatorname{acc}^{+}(X) \cap T$, we have $\bar{x} \subseteq \operatorname{Im}\left(f_{\alpha}\right)$. Pick a condition $q$ extending $p$, such that $\langle q(k) \mid \bar{\theta} \leq k<\bar{\theta}+\operatorname{otp}(\bar{x})\rangle$ forms the increasing enumeration of $\bar{x}$. Then $q$ forces that $\epsilon \in C_{\alpha} \backslash \varepsilon$ and $\operatorname{succ}_{\sigma}\left(C_{\alpha} \backslash \epsilon\right) \subseteq X$.

In $V$, by $\mathrm{CH}_{\lambda}$ and the main result of [She10], $\nabla(\kappa)$ holds. By $\lambda^{<\theta}=\lambda<\kappa, \operatorname{Col}(\theta, \lambda)$ is a $\kappa-c c$ poset of size $<\kappa$, so that the same argument from the proof of Theorem 3.4 yields that in $V[G]$, there exists a sequence $\left\langle Z_{\alpha} \mid \alpha<\kappa\right\rangle$ satisfying that for every $A \in \mathcal{P}^{V[G]}(\kappa)$, there exists some $X_{A} \in \mathcal{P}^{V}(\kappa)$ such that:

(1) $V \models X_{A}$ is stationary;

(2) $V[G] \models X_{A} \subseteq\left\{\beta<\lambda^{+} \mid Z_{\beta}=A \cap \beta\right\}$.

As $\left(\theta^{+}\right)^{V[G]}=\left(\lambda^{+}\right)^{V}=\kappa$, and as $\operatorname{Col}(\theta, \lambda)$ is a $\kappa$-cc poset, stationary subsets of $\kappa$ from $V$ remain stationary in $V[G]$, so that $V[G] \models \diamond(\kappa)$.

Work in $V[G]$. For every $\alpha \in T$, let $C_{\alpha}^{\bullet}:=\operatorname{Im}\left(g_{\alpha}\right)$, where $g_{\alpha}: C_{\alpha} \rightarrow \alpha$ is defined exactly as in the proof of Theorem 3.4 (page 11). Then $C_{\alpha}^{\bullet}$ is again a club in $\alpha$ of order-type $\theta$.

Claim 3.6.2. For every cofinal subset $A \subseteq \kappa$ (from $V[G]$ ), there exists a club $E_{A} \subseteq \kappa$, such that for every $\alpha \in T \cap E_{A}$ and every $\sigma<\theta$, we have $\sup \left\{\eta \in C_{\alpha}^{\bullet} \mid \operatorname{succ}_{\sigma}\left(C_{\alpha}^{\bullet} \backslash \eta\right) \subseteq A\right\}=\alpha$.

Proof. The result follows from Claim 3.6.1 just like Claim 3.4.5 follows from Claim 3.4.4.

For every $\alpha \in E_{\theta}^{\kappa} \backslash T$, let $C_{\alpha}^{\bullet}$ be an arbitrary club subset of $\alpha$ of order-type $\theta$.

\footnotetext{
${ }^{8}$ Let us point out that $C_{\lambda}$ witnesses the crucial reason for $\operatorname{Col}(\theta, \lambda) \in \mathbb{C}_{\lambda}$. That is, by taking $\Lambda:=C_{\lambda}$, we get that $\Lambda$ is a club in $\lambda$ of order-type $\operatorname{cf}(\lambda)$ such that for every function $f \in{ }^{\lambda} \lambda \cap V$, there exists some $\xi \in \Lambda$ with $f(\xi)<\min (\Lambda \backslash(\xi+1))$. Indeed, given $f \in{ }^{\lambda} \lambda \cap V$, simply pick $X \subseteq \kappa$ from $V$ such that $X \backslash \lambda=\kappa \backslash \lambda$, and such that $X \cap \lambda$ is a cofinal subset of $\lambda$ satisfying that for all $\xi \in X \cap \lambda, f(\xi)<\min (X \backslash(\xi+1))$. Now, appeal to our claim with this $X$ and with $\sigma=3$.
} 
Finally, for every $\alpha<\kappa$, let:

$$
\mathcal{C}_{\alpha}^{\bullet}:= \begin{cases}\{\emptyset\}, & \text { if } \alpha=0 ; \\ \{\{\beta\}\}, & \text { if } \alpha=\beta+1 ; \\ \left\{C_{\alpha}^{\bullet}\right\}, & \text { if } \alpha \in E_{\theta}^{\kappa} ; \\ \left\{c \in[\alpha]^{<\theta} \mid c \text { is a club in } \alpha\right\}, & \text { otherwise. }\end{cases}
$$

As $V \models \lambda^{<\theta}=\lambda$, and $\operatorname{Col}(\theta, \lambda)$ is $(<\theta)$-closed, we have $\left|[\lambda]^{<\theta}\right| \leq|\lambda|=\theta$, so that $\left|\mathcal{C}_{\alpha}^{\bullet}\right|<\kappa$ for all $\alpha \in E_{<\theta}^{\kappa}$. Altogether, $\left\langle\mathcal{C}_{\alpha}^{\bullet} \mid \alpha<\kappa\right\rangle$ witnesses $\mathrm{P}^{*}(T, \theta)$.

Proposition 3.7. Suppose that $\lambda^{<\lambda}=\lambda$ is an infinite cardinal, and $\kappa>\lambda$ is a strongly inaccessible cardinal. If $\mathbb{P}$ is a $(<\lambda)$-distributive, $\kappa$-cc notion of forcing, collapsing $\kappa$ to $\lambda^{+}$, then $V^{\mathbb{P}} \models \mathrm{P}^{*}\left(E_{\lambda}^{\lambda^{+}}, \lambda\right)+\diamond\left(\lambda^{+}\right)$.

Proof. As $\kappa$ is strongly inaccessible, for every $\alpha<\kappa$, the collection $\mathcal{N}_{\alpha}:=\left\{\tau \in V_{\alpha+1} \mid \tau\right.$ is a $\mathbb{P}$-name $\}$ has size $<\kappa$. Let $G$ be $\mathbb{P}$-generic over $V$, and work in $V[G]$. For every $\mathbb{P}$-name $\tau$, denote by $\tau_{G}$ its interpretation by $G$. Then, for all $\alpha<\kappa$, let $\mathcal{A}_{\alpha}:=\left\{\tau_{G} \mid \tau \in \mathcal{N}_{\alpha}\right\} \cap \mathcal{P}(\alpha)$. Since $\mathbb{P}$ has the $\kappa$-cc, $\left\langle\mathcal{A}_{\alpha} \mid \alpha<\kappa\right\rangle$ forms a $\diamond^{+}\left(\lambda^{+}\right)$-sequence, and in particular, $\diamond^{*}\left(E_{\lambda}^{\lambda^{+}}\right)$and $\diamond\left(\lambda^{+}\right)$hold. Since $\mathbb{P}$ is $(<\lambda)$-distributive, we still have $\lambda^{<\lambda}=\lambda$. Finally, by [BR16], $\diamond^{*}\left(E_{\lambda}^{\lambda^{+}}\right)+\lambda^{<\lambda}=\lambda$ entails $\mathrm{P}^{*}\left(E_{\lambda}^{\lambda^{+}}, \lambda\right)$.

\section{REFERENCES}

[AS83] Uri Abraham and Saharon Shelah. Forcing closed unbounded sets. J. Symbolic Logic, 48(3):643-657, 1983.

[AAK16] Domink Adolf, Arthur W. Apter, and Peter Koepke. Singularizing Successor Cardinals by Forcing. Submitted, 2016.

[BR15a] Ari Meir Brodsky and Assaf Rinot. A microscopic approach to Souslin-tree constructions. Part I. arXiv preprint arXiv:1601.01821, 2015.

[BR15b] Ari Meir Brodsky and Assaf Rinot. Reduced powers of Souslin trees. arXiv preprint arXiv:150\%.05651, 2015.

[BR16] Ari Meir Brodsky and Assaf Rinot. A microscopic approach to Souslin-tree constructions. Part II. in preparation, 2016.

[CFM01] James Cummings, Matthew Foreman, and Menachem Magidor. Squares, scales and stationary reflection. J. Math. Log., 1(1):35-98, 2001.

[CS02] James Cummings and Ernest Schimmerling. Indexed squares. Israel J. Math., 131:61-99, 2002.

[DJ74] Keith J. Devlin and Hȧvard Johnsbräten. The Souslin problem. Lecture Notes in Mathematics, Vol. 405. Springer-Verlag, Berlin, 1974.

[DS95] Mirna Džamonja and Saharon Shelah. On squares, outside guessing of clubs and $I_{<f}[\lambda]$. Fund. Math., 148(2):165-198, 1995.

[FH16] Laura Fontanella and Yair Hayut. Square and Delta reflection. Ann. Pure Appl. Logic, 167(8):663-683, 2016.

[Git95] Moti Gitik. Some results on the nonstationary ideal. Israel J. Math., 92(1-3):61-112, 1995.

[Gre76] John Gregory. Higher Souslin trees and the generalized continuum hypothesis. J. Symbolic Logic, 41(3):663671, 1976.

[Jen72] R. Björn Jensen. The fine structure of the constructible hierarchy. Ann. Math. Logic, 4:229-308; erratum, ibid. 4 (1972), 443, 1972. With a section by Jack Silver.

[LH16] Chris Lambie-Hanson. Aronszajn trees, square principles, and stationary reflection. arXiv preprint arXiv:1605.05489, 2016.

[Rin09] Assaf Rinot. A cofinality-preserving small forcing may introduce a special Aronszajn tree. Arch. Math. Logic, 48(8):817-823, 2009.

[Rin16] Assaf Rinot. Higher Souslin trees and the GCH, revisited. submitted, 2016.

[Sch05] Ernest Schimmerling. A question about Suslin trees and the weak square hierarchy. Notre Dame J. Formal Logic, 46(3):373-374 (electronic), 2005. 
[She10] Saharon Shelah. Diamonds. Proceedings of the American Mathematical Society, 138:2151-2161, 2010. arxiv:0711.3030.

[Spe49] E. Specker. Sur un problème de Sikorski. Colloquium Math., 2:9-12, 1949.

[Tod84] S. Todorčević. Trees and linearly ordered sets. In Handbook of set-theoretic topology, pages 235-293. NorthHolland, Amsterdam, 1984.

[Tod87] Stevo Todorčević. Partitioning pairs of countable ordinals. Acta Math., 159(3-4):261-294, 1987.

Department of Mathematics, Bar-Ilan University, Ramat-Gan 5290002, Israel.

URL: http://u.math.biu.ac.il/ brodska/

$U R L:$ http://www.assafrinot.com 